Voix et Images

voixetimages

\title{
Solitaires dans la foule
}

\section{Gabriel Landry}

Volume 29, numéro 3 (87), printemps 2004

France Daigle

URI : https://id.erudit.org/iderudit/009230ar

DOI : https://doi.org/10.7202/009230ar

Aller au sommaire du numéro

\section{Éditeur(s)}

Université du Québec à Montréal

\section{ISSN}

0318-9201 (imprimé)

1705-933X (numérique)

Découvrir la revue

Citer ce compte rendu

Landry, G. (2004). Compte rendu de [Solitaires dans la foule]. Voix et Images, 29(3), 167-170. https://doi.org/10.7202/009230ar d'utilisation que vous pouvez consulter en ligne.

https://apropos.erudit.org/fr/usagers/politique-dutilisation/ 


\author{
P O ÉS I E \\ Solitaires dans la foule \\ $+++$
}

GABRIEL LANDRY

Collège de Maisonneuve

Bon sang de bonsoir! Un livre de poèmes qui frise les trois cents pages! L'amateur d'ordinaire tenu au régime des plaquettes tient sa revanche. Je veux dire sur ses amis et sa blonde, qui dévorent, au temps des vacances, à l'ombre du parasol ou sur la galerie du chalet, de toujours imposants romans. Un fort volume a quelque chose d'estival en soi. Mais la plaquette et la plage sont inconciliables. La plaquette appelle la chambre ou la cellule. Or, voilà un livre de poésie que le soleil ne desquamera pas, qu'on pourra déposer sur le sable sans qu'il parte au vent, qui nécessitera l'usage d'un signet, car deux cent quatre-vingt-quinze pages, tout de même, on ne lit pas ça d'une traite. Un livre qui fera donc de vous un lecteur anonyme parmi la foule lectrice des vacances de la construction.

Ainsi devisais-je bêtement, encore à l'étonnement d'avoir reçu Il y a quelqu'un ${ }^{1}$ ?, le nouveau titre d'Hélène Monette, qui pesait aussi lourd dans ma main que n'importe quelle grosse affaire de Marie Laberge. La dernière fois que le poème-grenouille s'était fait aussi gros que le roman-bœuf, ça remontait à Une certaine fin de siècle, du vaillant Claude Beausoleil, avec son refrain-avertissement désormais célèbre nous reviendrons comme des Nelligan. Je n'en étais pas revenu.

\title{
NO WOMAN'S LAND
}

Le monde qu'Hélène Monette donne à voir dans Il y a quelqu'un? est un monde dévasté, une planète de culs-de-sac et de chemins impraticables, un «monde sous zéro» (119). Topographie du désenchantement que précisent les intitulés des diverses sections du livre: «Impasse des Confettis», «Place du Déluge», «Autoroute du Système», "Ponts de glace», "Impasse des Splendeurs». La relative synonymie de ces titres suggère assez qu'on ne saurait, dans cet univers monochrome, changer le mal de place. Le motif récurrent du train, métonymie du voyage, bien sûr, mais aussi de la vie qui passe («et le train s'en vient/maudit train d'enfer», [115]), figure la fuite perpétuelle et l'absurdité de toute mobilité, car «nous avançons nowhere» (263) et: «il n'y a pas de gare/qu'un train fou dans la nuit» (116). Des quatre coins de cet univers où «il n'y a plus rien» (159), où «l'Arche de Nobody est une banque qui prend l'eau» (163), Monette élève une voix

$$
+++
$$

1 Hélène Monette, Il y a quelqu'un?, Montréal, Boréal, 2004, 295 p. 
prolixe où s'entremêlent plaintes et revendications, cris et chuchotements, appels d'air et miserere, élans lyriques et boutades décapantes.

Le copieux récitatif a quelque chose de paradoxal, car si, d'un côté, il cherche à exprimer l'absence, la solitude (amoureuse) ou le manque, de l'autre il vient saturer de paroles ce monde dépeuplé. On me dira qu'il s'agit d'un renversement calculé et symbolique, le chant du poème venant combler tous les vides. Je reste d'avis qu'on aurait dû presser davantage cette éponge gorgée d'eau. Ce n'est pas la longueur du texte qui est en cause, c'est sa redondance. Les infatigables attaques contre Big Brother, le Système, l'américanisme, si elles brillent souvent par un sens remarquable du décapant et du satirique, finissent, à force de redites, par se diluer. Elles ne sont pas sans verser, ça et là, dans un registre qu'il vaudrait sans doute mieux laisser aux poètes de brousse ou aux Francs-tireurs de Télé-Québec, ces grands singes de la Protestation. Dans Il y a quelqu'un?, en tout cas, la veine contestataire a tendance à réduire le poème à la nomenclature de ses cibles.

Je dis poème, je devrais dire poésie. Le livre donne en effet l'impression d'une seule et longue coulée prosaïque, dont les laisses plus ou moins interchangeables auraient été jointes pour composer un discours qui paraît sans sutures. La mention générique de la couverture est claire: poésie. C'est un singulier éloquent. Alors que tant d'autres colligent minutieusement des poèmes, Monette fait entendre sa voix, souffle jusqu'à épuisement dans l'harmonica du pathos. Monologisme où la subjectivité l'emporte au bout du compte sur la volonté de s'extérioriser par la critique du système. La posture (quel affreux mot) d'Hélène Monette est plus romantique qu'il n’y paraît. «Je déromantiserai/ce fantasme d'être ensemble» (118), dit-elle à propos de la relation amoureuse, mais tout son livre romantise la prise de parole (poétique, singulière, en marge) et la solitude d'un moi aussi vivant par l'écriture que possible. Un seul être vous manque et tout est dépeuplé, écrivit Lamartine. La sentence aurait pu figurer, aussi bien que les vers de la déréliction mironienne, en exergue d'un livre qui veut avant tout résister. Résister au cynisme, mais aussi à la séduction des glacis formels et stylistiques recouvrant parfois les poèmes, quand ils sont concoctés loin du monde, parcimonieusement, en toutes quiétude et compétence.

\section{FOULE SENTIMENTALE OU MON NOM EST PERSONNE}

Retour aux plaquettes. Ce n'est pas le nombre de pages qui m'a étonné quand j'ai reçu Vers quelque (sommes nombreux à être seul) ${ }^{2}$, c'est ce titre trouvé, presque pétard. J'ai spontanément songé à quelque roublardise d'un tire-à-la-ligne formaliste, étant donné le double sens de vers. Vers quelque comme dans mètre quelconque, ou comme dans une nouvelle inversion transgressive, mais postulant la modestie et suggérant le gentil bouquet, de quelques vers. J'avais tout faux.

Hélène Monette se demande s'il y a quelqu'un. Danny Plourde lui répond. Il y a plein de monde, ça fourmille de solitudes toutes pareilles. Celle du poète n'est pas exceptionnelle, remettez donc dans vos cartables vos paroles par trop singulières, poètes, vous êtes seuls dans la foule des solitaires, et foin de l'enflure du moi: "ai choisi d'être comme tout le monde» (11). Pour que le lecteur ne se méprenne pas sur ses intentions fraternalistes, Danny Plourde a évincé le je de tous ses poèmes et nous

$$
++
$$

2 Danny Plourde, Vers quelque (sommes nombreux à être seul), Montréal, l’Hexagone, 2004, 92 p. 
informe, dans un liminaire instructif où il déplore l'élitisme pédant de ses pairs, du choix qu'il a fait d'écrire "pour tous ceux qui ne savent pas bien lire mais qui ont tout de même l'œil ouvert» et pour «sa mère qui n'a qu'un secondaire un». Je me suis demandé ce qui serait arrivé si Rimbaud avait choisi d'écrire pour sa mère. Baudelaire l'a fait, mais uniquement dans sa correspondance.

Passé l'irritation de la profession de foi populiste et anti-littéraire, bravade collégienne au reste désavouée dès la page 16, à mon contentement, par l'allusion plus que livresque à une certaine Nadja, et, disons-le, par une relative tenue de l'écriture au fil des pages, j'ai trouvé des qualités à ce premier livre d'un jeune poète. Un sens de l'image (c'est cette vertu, quand elle est au rendez-vous, qui sauve de l'échec total de nombreux projets d'écriture), une manière d'emprunter des raccourcis inopinés, une gouaille, une raillerie qui sont moins faciles qu'on pourrait le croire. Mais ces qualités sont diablement enveloppées, prises dans la gangue d'un prêchi-prêcha politico-quelque chose, vers quoi s'en vont bon nombre de ces poèmes engagés sur la pente du questionnement identitaire:

\author{
mais n'être rien \\ n'être personne n'être nulle part (28).
}

Questionnement ou affirmation? Rhétorique assurément rédhibitoire, en tout cas, ces vers plus que quelque:

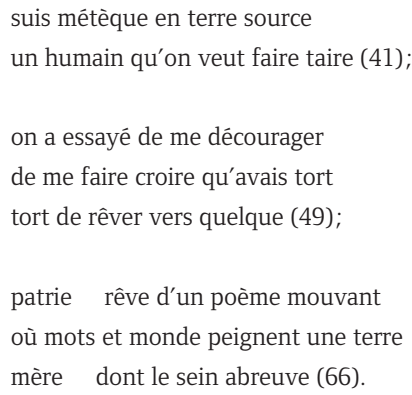

Je ne dirai rien du dernier ensemble où la devise Je me souviens donne lieu à un sentimentalisme collectiviste qui ferait applaudir tous les cégépiens souverainistes dans une soirée culturelle. Dans son premier recueil, dit l'éditeur, Danny Plourde révèle la force poétique d'une parole affranchie de son «je». Parions sur le deuxième recueil pour un affranchissement total et salutaire du «nous». À raison d'une plaquette par an et compte tenu du nombre assez restreint des pronoms personnels, l'auteur devrait, dans un proche avenir, conquérir contre ces tyranneaux son entière autonomie.

\title{
PERSONNE DU PLUS GRAND NOMBRE
}

Quand j'ai lu le titre du dernier livre de Pierre Desruisseaux ${ }^{3}$, bon sang de bonsoir, je me suis demandé si notre vétéran et la recrue Plourde ne s'étaient pas donné le mot. La

$$
+++
$$

3 Pierre Desruisseaux, Personne du plus grand nombre, Montréal, l'Hexagone, 2003, 69 p. 
collection "L'Appel des mots», justement, dirigée par Robbert Fortin, portait-elle son nom au point que les poètes s'y répondissent d'un intitulé à l'autre? Mais la lecture des poèmes de Personne du plus grand nombre m'a signifié assez tôt que la parenté des titres n'est que la parenté des titres.

Il y a pourtant aussi, chez Desruisseaux, cette modulation à partir de l'identité du poète, homme de paroles et d'une mémoire qui ne lui appartiennent pas en propre, et cet élan de soi vers l'autre qui lui fait qualifier un de ses poèmes de prolétarien (20). Cependant l'effacement du moi est ici plus effectif, car il s'agit de

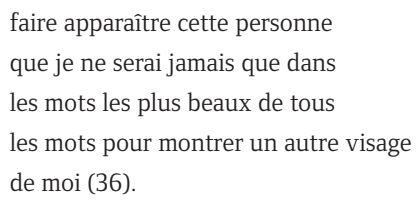

Desruisseaux propose une méditation sur la figure du poète qu'on pourrait rapprocher de l'exigence d'un Jaccottet («L'effacement soit ma façon de resplendir») et qui s'intéresse, d'une manière plus générale, au poète en situation dans les plis de l'histoire ou les aléas du quotidien. La rencontre ou le côtoiement des semblables frères, peut-être lecteurs mais surtout itinérants, exclus, pêcheurs, prêtres solitaires, écoliers, tous habitants d'un monde sans foi ni loi, confère à cette méditation ses accents les plus empathiques. La compassion s'impose, dans laquelle se profile un christianisme discret. Le poète n'est pas seul, si ce n'est devant le silence et la mort - comme le plus grand nombre.

\section{JE SUIS UN POÈTE}

Croyez-le ou non, lisant Professeur de poésie ${ }^{4}$, le je ne sais plus combientième titre d'André Roy, qui en a publié beaucoup, j'ai songé à L'Épître aux Pisons, l'art poétique $\mathrm{d}^{\prime}$ Horace, un poète assez peu herberougiste. Un lecteur n'a pas toujours le choix de ses raccords, non plus qu'un chroniqueur celui de ses raccourcis.

Prescriptions, considérations sur la conduite du poète dans la vie et dans l'écriture, appels du maître à l'élève, c'est bel et bien un art poétique que composent la cinquantaine de poèmes proposés ici. Les lecteurs qui n'ont pas ouvert un livre de Roy plus récent que ceux qu'il écrivait aux belles heures de ses contorsions formalistiques s'étonneront de cette lisibilité nouvelle et remarquable qu'il cultive. La ponctuation, que le vers-librisme a reléguée au rang des vieilleries, est au rendez-vous. La syntaxe est des plus claires et obéit presque toujours au principe classique de sa coïncidence avec le vers. Chaque morceau a la sobriété d'une épigramme méditant sur la jouissance d'écrire ou la fragilité de toute postérité. Le professeur est venu tempérer la singularité présumée du moi poétique, et c'est à son regard critique qu'on doit sans doute cette distance prise avec un ancien moi («le spécialiste du "je" » [46]), mais c'est à n'en pas douter un poète qui parle ici de son «écriture célibataire» (39). Pour magnifier la poésie, «langue déclarée morte» (24), et les poètes de tous les temps, «homme[s] de bien» (64) jamais tout à fait seuls, puisqu'ils donnent «de la poésie à ceux qui n'en ont pas» (30).

$$
+++
$$

4 André Roy, Professeur de poésie, Montréal, Les Herbes rouges, 2003, 71 p. 